\title{
Correlation of ultrasonography, hysterosalpingography and hystero- laparoscopy findings in cases of infertility
}

\author{
Falahunisa S. Shaikh*
}

Department of Obstetrics and Gynecology, Indian Naval Hospital Asvini, Mumbai, Maharashtra, India

Received: 20 September 2017

Accepted: 27 October 2017

\section{*Correspondence:}

Dr. Falahunisa S. Shaikh,

E-mail: shaikh_falah@yahoo.com

Copyright: (C) the author(s), publisher and licensee Medip Academy. This is an open-access article distributed under the terms of the Creative Commons Attribution Non-Commercial License, which permits unrestricted non-commercial use, distribution, and reproduction in any medium, provided the original work is properly cited.

\begin{abstract}
Background: Infertility is a condition which has medical as well as social impact on the state of the couple. Approximately 10-15\% couples are infertile. A wide arena of tests is available for the diagnosis of the causes of infertility. The aim of present study is to correlate the findings of ultrasonography, hysterosalpingography and hysterolaparoscopy in infertility.

Methods: It is a prospective study done over 2 years at INHS Asvini hospital, Mumbai. Infertile women underwent ultrasonography, hysterosalpingography and hystero-laparoscopy. Data was assessed using SPSS 17 software.

Results: Of 105 patients, on ultrasonography, 22.9\% had Polycystic ovaries (PCO), 2.9\% had ovarian cyst, $4.8 \%$ had fibroid, $4.8 \%$ had endometrioma, 3.8\% had adnexal mass, 3.8\% had polyp. On hysterosalpingography, 18 (17.1\%) had left and 20 (19\%) had right tubal block, $11(10.47 \%)$ had B/L tubal block, $6(5.7 \%)$ had septate uterus, $6(5.7 \%)$ had hydrosalpinx. On laparoscopy, 12 (11.4\%) had PCO, 4 (3.8\%) had ovarian cyst, $12(11.4 \%)$ had fibroid, 7 (6.7\%) had endometrioma, $19(18.1 \%)$ had left and $18(17.1 \%)$ had right tubal block, $12(11.42 \%)$ had $\mathrm{B} / \mathrm{L}$ tubal block, 4 (3.8\%) had hydrosalpinx. On hysteroscopy, 7 (6.7\%) had septate uterus, no polyp seen.

On laparoscopy, 21 (20\%) had endometriosis, 1 (1.9\%) had bicornuate uterus.

Conclusions: On comparing the results it was observed that hysterolaparoscopy gives definitive diagnosis and should be considered gold standard, though other modalities of investigation should be considered complimentary and not competing with it, as they are noninvasive.
\end{abstract}

Keywords: Hysterosalpingography, Hysteroscopy, Infertility, Laparoscopy, Ultrasonography

\section{INTRODUCTION}

Infertility is a condition which has medical as well as social impact on the state of the couple. Infertility means inability to conceive within one year of unprotected regular sexual intercourse, further classified as primary infertility, in which no previous pregnancies have occurred and secondary infertility, in which a prior pregnancy, although not necessarily a live birth, has occurred. Infertility affects approximately $10-15 \%$ of couples and is an important part of clinical practice for gynecologist. ${ }^{1}$
An optimal initial infertility investigation protocol would be a process that is diagnostically accurate, expeditious, cost-effective, reliable and as minimally invasive as possible.

In addition, the investigation should provide the clinician with useful prognostic information regarding possible future treatment. A wide arena of tests are available for the diagnosis of infertility. It is more important to perform the relevant investigation in a logical order at a correct time rather than performing routine tests in a series. 
In the present study our aim is to correlate the findings of USG (ultrasonography), HSG (hysterosalpingography) and hysterolaparoscopy in infertile patients.

Ultrasound helps in visualization of the uterine cavity, the endometrial thickness, any abnormality like polyp, myoma, or uterine septum, ovarian cysts, fallopian tubes is not routinely seen, unless if there is hydrosalpinx. Nowadays with the advent of 3- dimensional ultrasound machines, USG has become more efficacious in detecting pathologies.

Hysterosalpingography (HSG) is a radiologic procedure in which radio opaque dye is inserted in the uterine cavity. It is mainly used to assess the tubal patency and also shows the general configuration of the uterine cavity.

Advances in endoscopic surgery have revolutionized the approaches of gynaecologist for diagnosis and management of patients with infertility. Diagnostic laparoscopy is generally not a part of initial infertility evaluation; however, number of reports have shown that it is effective procedure for evaluation of long- term, unexplained infertility. Laparoscopy provides information regarding tubal and ovarian status, tubal patency, uterine configuration, pelvic pathology e.g. pelvic inflammatory disease, endometriosis, pelvic congestion and tuberculosis.

\section{METHODS}

This is an observational prospective study done in a INHS Asvini hospital, Mumbai over a duration of 2 years. Infertile women willing for work up were included in the study.

Couples who had not lived together for at least 12 months and patients with absolute or relative contraindication for laparoscopy i.e. any pre-existing cardiovascular or respiratory condition, generalized peritonitis, intestinal ileus or obstruction and abdominal hernia, were excluded. Sample size taken was 105.

Infertile women who gave consent were first subjected to a detailed history followed by clinical examination. Routine investigations include hemoglobin percentage, blood group and Rh type, HIV, HbsAg, VDRL, Anti $\mathrm{HCV}$, urine routine examination, electrocardiogram and chest X-ray.

- Transvaginal ultrasonography was done on day - 9 of the menstrual cycle with 2- D Siemens ultrasound machine.

- Hysterosalpingography was done between 8-10 days of menstrual cycle. Under fluoroscopic guidance contrast media $3-5 \mathrm{ml}$ (Urograffin 60\%) is injected to delineate uterine cavity. $\mathrm{X}$ ray films were taken as tubes begin to fill and when peritoneal spill has occurred.
- Hysterolaparoscopy was performed in the post menstrual phase under general anaesthesia. Hysteroscopy was performed with a Karl Storz 2.9 $\mathrm{mm}$ hysteroscope and the distending medium used is normal saline. Thereafter laparoscopy was done. The uterus, bilateral ovaries and tubes, pouch of douglas, ovarian fossa and upper abdomen was assessed. Chromopertubation was performed by inserting methylene blue dye under vision.

\section{Statistical analysis}

All the data was collected on a predesigned proforma and was entered in Microsoft Excel sheet. It was assessed by SPSS ver. 17 software for statistical analysis. P-value of $<0.05$ was considered as significant.

\section{RESULTS}

Patients were studied as regards their demographic characteristics, ultrasonography, hysterosalpingography and hysterolaparoscopy findings and also the results were compared.

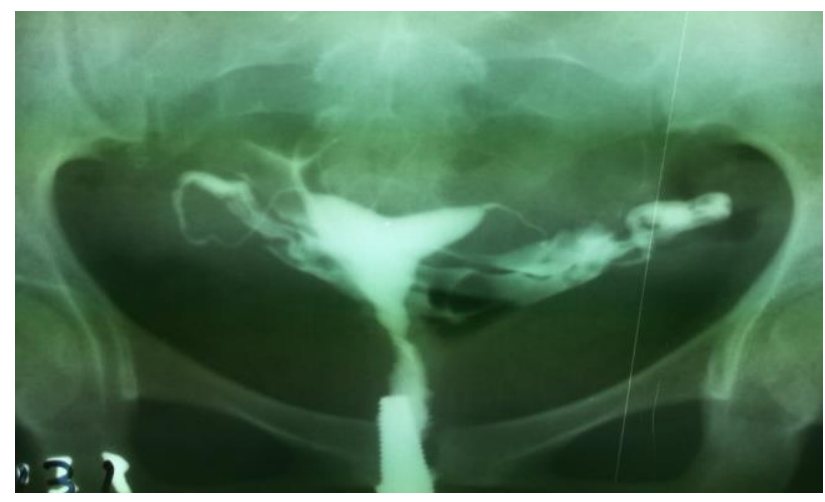

Figure 1: Subseptate uterus on HSG which was confirmed on hysterolaparoscopy.

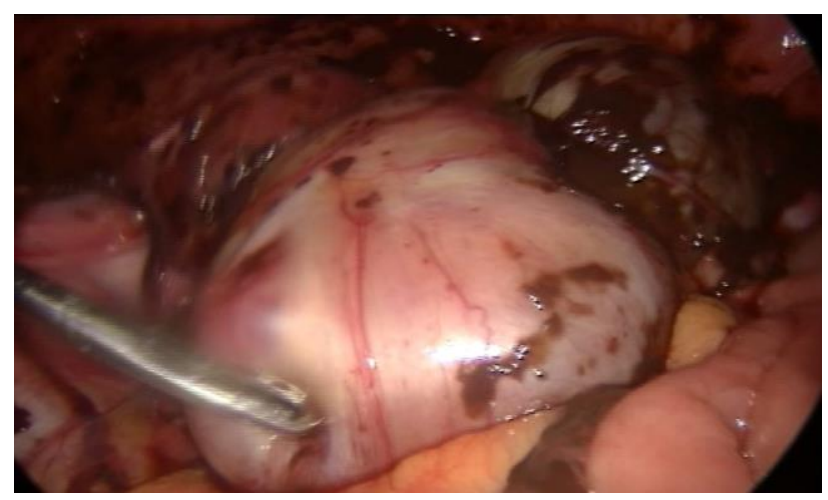

Figure 2: Subseptate uterus on HSG which was confirmed on hysterolaparoscopy.

Out of total 105 patients, $82(78.1 \%)$ were primary infertility and $23(21.9 \%)$ were secondary infertility. On analyzing the age wise distribution of the patients, mean age of the patients were $26.6 \mathrm{yrs} \pm 3.0$ with minimum age 
of 21 yrs and maximum age of 35 yrs. Also, maximum number of patients of primary $(52.4 \%)$ and secondary $(56.5 \%)$ infertility fall in the age group of 26-30 yrs. Duration of infertility in present study ranged from 1- 15 yrs. Mean duration of infertility was $4.28 \mathrm{yrs} \pm 2.7$, of which mean in primary infertility was 3.58 yrs and 6.7 yrs in secondary infertility. Maximum number of patients of primary infertility had duration of infertility of $<5 \mathrm{yrs}$ and maximum number of patients of secondary infertility had duration of infertility in the range of 5-10 yrs.

Table 1: Comparison of the findings on ultrasonography and laparoscopy.

\begin{tabular}{|c|c|c|c|c|c|}
\hline \multirow{2}{*}{ USG } & & \multicolumn{2}{|c|}{ Laproscopy } & \multirow{2}{*}{ Total } & \multirow{2}{*}{ p-value } \\
\hline & & No & Yes & & \\
\hline \multirow{6}{*}{$\mathrm{PCO}$} & No & 77 & 4 & 81 & \multirow{6}{*}{$<0.001$} \\
\hline & $\%$ & $95.10 \%$ & $4.90 \%$ & $77.1 \% \%$ & \\
\hline & Yes & 16 & 8 & 24 & \\
\hline & $\%$ & $66.70 \%$ & $33.30 \%$ & 22.9 & \\
\hline & Total & 93 & 12 & 105 & \\
\hline & $\%$ & $88.60 \%$ & $11.40 \%$ & $100.00 \%$ & \\
\hline \multirow{6}{*}{ Fibroid } & No & 91 & 9 & 100 & \multirow{6}{*}{$<0.01$} \\
\hline & $\%$ & $91.00 \%$ & $9.00 \%$ & $95.2 \%$ & \\
\hline & Yes & 2 & 3 & 5 & \\
\hline & $\%$ & $40.00 \%$ & $60.00 \%$ & $4.8 \% \%$ & \\
\hline & Total & 93 & 12 & 105 & \\
\hline & $\%$ & $88.60 \%$ & $11.40 \%$ & $100.00 \%$ & \\
\hline \multirow{6}{*}{ Ovarian Cyst } & No & 100 & 2 & 102 & \multirow{6}{*}{$<0.001$} \\
\hline & $\%$ & $98.00 \%$ & $2.00 \%$ & $97.2 \%$ & \\
\hline & Yes & 1 & 2 & 3 & \\
\hline & $\%$ & $33.30 \%$ & $66.70 \%$ & $2.9 \%$ & \\
\hline & Total & 101 & 4 & 105 & \\
\hline & $\%$ & $96.20 \%$ & $3.80 \%$ & $100.00 \%$ & \\
\hline \multirow{6}{*}{ Endometrioma } & No & 97 & 3 & 100 & \multirow{6}{*}{$<0.001$} \\
\hline & $\%$ & $97.00 \%$ & $3.00 \%$ & $95.2 \%$ & \\
\hline & Yes & 1 & 4 & 5 & \\
\hline & $\%$ & $20.00 \%$ & $80.00 \%$ & $4.8 \% \%$ & \\
\hline & Total & 98 & 7 & 105 & \\
\hline & $\%$ & $93.30 \%$ & $6.70 \%$ & $100.00 \%$ & \\
\hline
\end{tabular}

Table 2: Comparison in the findings on HSG and laparoscopy.

\begin{tabular}{|c|c|c|c|c|c|}
\hline \multirow{2}{*}{ HSG } & & \multicolumn{2}{|c|}{ Laproscopy } & \multirow{2}{*}{ Total } & \multirow{2}{*}{ p-value } \\
\hline & & No & Yes & & \\
\hline \multirow{6}{*}{ Left Tubal Block } & No & 77 & 10 & 87 & \multirow{6}{*}{$<0.001$} \\
\hline & $\%$ & $88.50 \%$ & $11.50 \%$ & $82.90 \%$ & \\
\hline & Yes & 9 & 9 & 18 & \\
\hline & $\%$ & $50.00 \%$ & $50.00 \%$ & $17.10 \%$ & \\
\hline & Total & 86 & 19 & 105 & \\
\hline & $\%$ & $81.90 \%$ & $18.10 \%$ & $100.00 \%$ & \\
\hline \multirow{6}{*}{ Right Tubal Block } & No & 78 & 7 & 85 & \multirow{6}{*}{$<0.01$} \\
\hline & $\%$ & $91.80 \%$ & $8.20 \%$ & $81.00 \%$ & \\
\hline & Yes & 9 & 11 & 20 & \\
\hline & $\%$ & $45.00 \%$ & $55.00 \%$ & $19.00 \%$ & \\
\hline & Total & 87 & 18 & 105 & \\
\hline & $\%$ & $82.90 \%$ & $17.10 \%$ & $100.00 \%$ & \\
\hline \multirow{6}{*}{ Hydrosalpinx } & No & 98 & 1 & 99 & \multirow{6}{*}{$<0.001$} \\
\hline & $\%$ & $99.00 \%$ & $1.00 \%$ & $94.30 \%$ & \\
\hline & Yes & 3 & 3 & 6 & \\
\hline & $\%$ & $50.00 \%$ & $50.00 \%$ & $5.70 \%$ & \\
\hline & Total & 101 & 4 & 105 & \\
\hline & $\%$ & $96.20 \%$ & $3.80 \%$ & $100.00 \%$ & \\
\hline
\end{tabular}


Out of the 45 patients with abnormal findings on USG, 24 had polycystic ovaries $(22.9 \%)$ which was the most common finding. The other findings were fibroid uterus in $5(4.8 \%)$, ovarian cyst in $3(2.9 \%)$, adnexal mass in 4 $(3.8 \%)$, endometrioma in $5(4.8 \%)$, uterine polyp in 4 $(3.8 \%)$ patients.

Out of the 55 patients with abnormal findings on hysterosalpingography, most common finding was tubal block. 11 patients $(10.47 \%)$ had bilateral tubal block, left tubal block in $18(17.1 \%)$, right tubal block in $20(19 \%)$ patients. The other findings were intra uterine filling defects in 4 patients $(3.8 \%)$, extravasation in $1(1 \%)$ patient, intra uterine septum in $6(5.7 \%)$ patients and hydrosalpinx in $6(5.7 \%)$ patients.

Table 3: Comparison of the findings on HSG and hysteroscopy.

\begin{tabular}{|c|c|c|c|c|c|}
\hline \multirow{2}{*}{ HSG } & & \multicolumn{2}{|c|}{ Hysteroscopy } & \multirow{2}{*}{ Total } & \multirow{2}{*}{ p-value } \\
\hline & & No & Yes & & \\
\hline \multirow{6}{*}{$\begin{array}{l}\text { Septate } \\
\text { uterus }\end{array}$} & No & 97 & 2 & 99 & \multirow{6}{*}{$<0.001$} \\
\hline & $\%$ & 99.0 & 28.6 & 94.3 & \\
\hline & Yes & 1 & 5 & 6 & \\
\hline & $\%$ & 1.0 & 71.4 & 5.7 & \\
\hline & Total & 98 & 7 & 105 & \\
\hline & $\%$ & 100.0 & 100.0 & 100.0 & \\
\hline
\end{tabular}

On hysteroscopy, 7 patients $(6.7 \%)$ had septum and in 3 (2.9\%) ostia was not seen. One patient had bicornuate uterus confirmed on laparoscopy.

On laparoscopy 63 patients $(60 \%)$ had abnormal finding. Most common finding was endometriosis in 21 patients (20\%). The other findings were B/L tubal block in 12 $(11.42 \%)$ patients, left tubal block in $19(18.1 \%)$ and right tubal block in $18(17.1 \%)$ patients, pelvic adhesions in $13(12.4 \%)$, fibroid in $12(11.4 \%)$, PCO in $12(11.4 \%)$, endometrioma in $7(6.7 \%)$, ovarian cyst in $4(3.8 \%)$, hydrosalpinx in $4(3.8 \%)$ patients. One patient had bicornuate and arcuate uterus each.

\section{DISCUSSION}

In everyday clinical practice, it is not always clear if and when exactly in the fertility work-up a diagnostic laparoscopy should be offered. There is a need for more randomized controlled trials to answer remaining questions regarding its value in the diagnosis and treatment of patients with infertility.

In present study 105 patients were assessed, out of which 82 cases $(78.1 \%)$ were of primary infertility and 23 cases $(21.9 \%)$ of secondary infertility, which correlates with the studies conducted by Jani RS et al $74 \%$ and $26 \%$, Kumar A et al $82 \%$ and $18 \%$ and $48 \%$ and $52 \%$, Shetty et al $68 \%$ and $32 \%$ for primary and secondary infertility respectively. ${ }^{2,3,6}$ In the present study, majority of the primary infertility patients belong to age group of 26-30 yrs (52.9\%) and secondary infertility to age group of 2630 yrs $(56.9 \%)$. Various studies like that of Shetty et al and Krishna $\mathrm{C}$ et al have shown that there is rise in age at which women present with infertility. ${ }^{4,6}$ In present study, $3(3.7 \%)$ patients of primary infertility and $6(26.1 \%)$ of secondary infertility were of age $>30$ years. Because of the decline in fertility and the increased time to conception that occurs after the age of 30 , women $>30$ years of age should be referred for infertility work-up after 6 months of trying to conceive. Importance of the age factor lies in the fact that as the age advances fertility decreases.

In the present study, majority of the patients of primary infertility $(91.5 \%)$ had a duration of $1-5$ years and that of secondary infertility $(52.2 \%)$ had duration of infertility $>5$ years. Duration of infertility ranged from 1- 15 yrs. Study conducted by Shetty et al and Gour et al correlates with the present study. ${ }^{5}$

Thus, majority of the infertile couple start worrying about their inability to conceive within 5 years of marriage and decide to get investigated.

Hysterosalpingography has been used for many years for the assessment of tubal patency. Laparoscopy enables direct visualisation of adnexa and to perform chromopertubation test (CPT) to assess tubal patency. Hysterosalpingography can also assess any intrauterine adhesions if there are any filling defects on radiographs, also extravasation with filling defects can be correlated with endometriosis. Any change in the shape of the uterine cavity on hysterosalpingography can be helpful towards diagnosis of any congenital malformation.

In a study conducted by $\mathrm{Zhu} \mathrm{H}$ et al, the sensitivity, specificity, positive predictive value and negative predictive value of TVS in the detection of endometrial polyps (EP) were 67, 96, 88.23 and 86.49\%, respectively. ${ }^{7}$ Compared with hysteroscopy as the gold standard, the sensitivity of TVS in detecting EP was relatively low; however, the two methods are similar in specificity, positive and negative prediction value. Though TVS may not replace hysteroscopy, it may be helpful to use TVS for initial screening of EP. A study by Yantapant examined $60 \mathrm{EP}$ patients with a mean age of 31-40 years. ${ }^{8}$ In that study, sensitivity, specificity and accuracy of the diagnosis of EPs by TVS were 60, 33.3 and $57.6 \%$, respectively. Vitner et al identified that, although hysteroscopy presented improved predictive values for diagnosing uterine polyps when compared with TVS, the difference was not statistically significant. ${ }^{9}$ A study by Babacan A et al concluded that for diagnosing any pathology, hysteroscopy had better specificity $(\mathrm{p}<0.001)$ although the two methods did not differ with regard to sensitivity $(\mathrm{p}=0.188) .{ }^{10}$

In a study conducted by Jain $\mathrm{P}$ et al the sensitivity of HSG is $93.3 \%$, specificity is $91.1 \%$, positive predictive value is $77.7 \%$ and negative predictive value is $97.6 \% .^{11}$ 
Lavy et al concluded that it is unnecessary to apply laparoscopy if HSG is normal or reveals suspicious unilateral tubal obstruction and therapy scheme does not alter in $95 \%$ of patients. ${ }^{12}$ However, laparoscopy is more beneficial for the patients with suspicious bilateral tubal pathology and alters therapy scheme. For tubal patency HSG was found to be satisfactory. However, some factors such as cornual spasm were held responsible for false positive tubal obstruction detected by HSG, while laparoscopy confirmed tubal patency for the same cases.

Table 4: Laparoscopy findings in comparison with other studies.

\begin{tabular}{|c|c|c|c|c|c|c|}
\hline Laparoscopy findings & Nayak et al ${ }^{13}$ & Gao $M^{14}$ & Shetty et $\mathrm{al}^{6}$ & Un Nisa $\mathbf{Z}^{15}$ & Parveen $\mathbf{S}^{16}$ & Present study \\
\hline Endometriosis & $37 \%$ & $18.75 \%$ & $10.7 \%$ & - & $19.35 \%$ & $20 \%$ \\
\hline Tubal occlusion & $31 \%$ & - & $26 \%$ & $25 \%$ & $16.12 \%$ & $11.42 \%$ \\
\hline Ovarian cyst & - & $8.05 \%$ & $4 \%$ & $2.5 \%$ & $4.8 \%$ & $3.8 \%$ \\
\hline Polycystic ovaries & - & $1.55 \%$ & $10 \%$ & - & $19.35 \%$ & $11.4 \%$ \\
\hline Myoma & $15 \%$ & $3.8 \%$ & $8 \%$ & $2.5 \%$ & $6.45 \%$ & $11.4 \%$ \\
\hline Pelvic adhesions & $26 \%$ & $59.3 \%$ & $13.75 \%$ & $7.5 \%$ & $11.2 \%$ & $12.4 \%$ \\
\hline Uterine anomaly & $3 \%$ & - & - & $7.5 \%$ & $12.9 \%$ & $1.9 \%$ \\
\hline
\end{tabular}

In our study, when the findings of hysterosalpingography were correlated with the findings on laparoscopy and it was found that out of the 18 patients who had left sided tubal block only 9 had similar findings on laparoscopy, whereas rest 10 new cases of left tubal block were seen on CPT. Out of the 20 cases of right sided tubal block on HSG, 11 corresponded on laparoscopy and 7 new cases had right tubal block on laparoscopy. 6 cases had hydrosalpinx on HSG of which only 3 were seen on laparoscopy and 1 new case of hydrosalpinx was seen on laparoscopy. The findings of septate uterus found on HSG were correlated with hysteroscopy findings and it was found that out of the 6 cases found to have septate uterus on HSG, 5 were confirmed on hysteroscopy and one patient was found to have bicornuate uterus which was confirmed on laparoscopy. 2 new cases were found to have septate uterus on hysteroscopy which was not seen on hysterosalpingography.

On hysteroscopy $7(6.7 \%)$ had septate uterus, $1(1.0 \%)$ had bicornuate uterus which was confirmed on laparoscopy and in $3(2.9 \%)$ patients ostia was not visualised which showed bilateral tubal block on laparoscopy. In a study by Nayak et al $10 \%$ had septum on hysteroscopy. ${ }^{17}$

As seen in the table above, most common finding seen on laparoscopy was endometriosis $(20 \%)$ seen in 21 patients in our study which correlated with the study by Nayak et al and Parveen S. ${ }^{13,16}$ Tubal occlusion was the most common cause in study conducted by Shetty et al and UnNisa Z. ${ }^{6,15}$

\section{CONCLUSION}

Although USG and HSG are generally done as the initial investigation for assessment of infertility, many a times especially in cases of long term unexplained infertility, positive findings may be missed. Also, as seen in our study HSG over diagnosis tubal block, may be due to uterine spasm induced by pain. At such times hysterolaparoscopy is gold standard. Also, endometriosis, pelvic adhesions, and uterine septum may be difficult to be picked up by routine imaging procedures. It should be appreciated that HSG and Laparoscopy are complimentary rather that competitive procedures. The accuracy of diagnosis is enhanced when two procedures are combined especially in those cases where the result of one of the tests is doubtful.

\section{Funding: No funding sources}

Conflict of interest: None declared

Ethical approval: The study was approved by the Institutional Ethics Committee

\section{REFERENCES}

1. Berek and Novak's Gynecology. Infertility and Assisted reproductive technology, $15^{\text {th }}$ ed. 2012;32:1134-64.

2. Jani RS, Munshi DS, Jani SK, Munshi SP. Application of laparoscopy in current fertility practice. Int J Reprod Contracept Obstet Gynecol. 2014;3:362-5.

3. Ashok Kumar K, Priyanka Jogi Y, Bharathi A. Diagnostic laparoscopy in the evaluation of female factor infertility. Int $\mathbf{J}$ Reprod Contracept Obstet Gynecol. 2017;6:383-7.

4. Krishna C, Prathima S, Chandraiah S, Anitha GS. Laparoscopy as a diagnostic tool in evaluation of female factors in infertility. Int J Reprod Contracept Obstet Gynecol. 2017;6:864-7.

5. Gour A, Zawiejska A, Mettler L. HysteroscopyCurrent trends and challenges. J Obstet Gynecol India. 2008;58(1):57-62.

6. Shetty SK, Shetty H. Diagnostic laparoscopy in infertility - A retrospective study. Int Study Biomed Res. 2013;04:07. 
7. Zhu H, Fu J, Lei H, Song Y, Shen L, Huang W. Evaluation of transvaginal sonography in detecting endometrial polyps and the pregnancy outcome following hysteroscopic polypectomy in infertile women. Experiment Therapeutic Med. 2016;12:1196-1200.

8. Yantapant A. Comparison of the accuracy of transvaginal sonography and hysteroscopy for the diagnosis of endometrial polyps at Rajavithi Hospital. J Med Assoc Thai. 2012;95Suppl3:S92S97.

9. Vitner D, Filmer S, Goldstein I, Khatib $\mathrm{N}$ and Weiner Z. A comparison between ultrasonography and hysteroscopy in the diagnosis of uterine pathology. Eur J Obstet Gynecol Reprod Biol. 2013;171:143-5.

10. Babacan A, Gun I, Kizilaslan C, Ozden O, Muhcu $\mathrm{M}$, Mungen E, et al. Comparison of transvaginal ultrasonography and hysteroscopy in the diagnosis of uterine pathologies. Int $\mathbf{J}$ Clin Exp Med 2014;7(3):764-769

11. Jain P, Bansal D, Deodhar P. Re-emerging role of HSG Vs laparoscopy for infertility work-up at rural hospital set up. J Res Med Dental Sci. 2015 Oct 1;3(4):287-9.
12. Lavy, Lev-Sagie A, Holtzer H, Revel A, Hurwitz A. Should laparoscopy be a mandatory component of the infertility evaluation in infertile women with normal hysterosalpingogram or suspected unilateral distal tubal pathology. Eur J Obstet Gynecol Reprod Biol. 2004;114(1):64-8.

13. Prasanta K Nayak, Purna C Mahapatra, JJ Mallack, Et al, Role of diagnostic hysteron laparoscopy in infertility: a retrospective study of 300 infertile patients. J Human Reprod Sci. 2013;6(1):32-34.

14. Gao M. Analysis of 188 Cases of Laparoscopic Diagnosis of Infertility. IJEME. 2013 Feb 29;3(2):1.

15. Butt ZU, Khan GH. Study of Infertility in Females by Laparoscopy in Remote Area. JRMC. 2009;13(2):89-91.

16. Parveen S. Study of combined diagnostic laparoscopy and hysteroscopy in infertile patients. $\mathbf{J}$ Surg Pak. 2010;15(1).

Cite this article as: Shaikh FS. Correlation of ultrasonography, hysterosalpingography and hysterolaparoscopy findings in cases of infertility. Int $\mathbf{J}$ Reprod Contracept Obstet Gynecol 2017;6:5390-5. 